\title{
28 Research Square \\ Ultrafast and accurate 16S rRNA microbial community analysis using Kraken 2 and Bracken
}

Jennifer Lu

Steven L. Salzberg

\section{Video Byte}

Keywords: Microbiome, $16 \mathrm{~S}$ ribosomal RNA sequencing, bacteria, Quantitative Insights Into Microbial Ecology, QIIME, metagenomics, Kraken 2, Bracken, q2-feature-classifier, Greengenes, SILVA, RDP, MAPseq, mothur, QIIME 2, database generation, classification, RAM, accuracy, alignment-free algorithm

Posted Date: November 12th, 2020

DOl: https://doi.org/10.21203/rs.3.rs-106644/v1

License: (c) (1) This work is licensed under a Creative Commons Attribution 4.0 International License.

Read Full License 


\section{Abstract}

Since the 1970s, 16S ribosomal RNA sequencing has been the primary means of identifying bacteria in environmental samples of unknown composition. Among the most powerful tools for this sequencing is the Quantitative Insights Into Microbial Ecology (QIIME) package whose newest release, QIIME 2, boasts one of the highest classification accuracies for simulated metagenomes from the human gut, ocean, and soil. But a recent study suggests that even more powerful tools are available namely Kraken 2 and Bracken. Compared with QIIM2, Kraken 2 and Bracken were up to 100 times faster in generating the wellknown Greengenes and SILVA 16S rRNA databases and up to 300 times faster at classification while using 100 times less RAM. Overall, Kraken 2 and Bracken produced more accurate 16S rRNA profiling results than QIIME2. Extremely fast, efficient, and accurate, Kraken 2 and Bracken could provide robust solutions for identifying and classifying bacterial species from a variety of environmental samples. 